\title{
Breastfeeding Practices, Demographic Variables, and Their Association with Morbidities in Children
}

\author{
Dipen V. Patel, ${ }^{1}$ Satvik C. Bansal, ${ }^{1}$ Archana S. Nimbalkar, ${ }^{2}$ Ajay G. Phatak, ${ }^{3}$ \\ Somashekhar M. Nimbalkar, ${ }^{1}$ and Rajendra G. Desai ${ }^{3}$ \\ ${ }^{1}$ Department of Pediatrics, Pramukhswami Medical College, Karamsad, Gujarat 388325, India \\ ${ }^{2}$ Department of Physiology, Pramukhswami Medical College, Karamsad, Gujarat 388325, India \\ ${ }^{3}$ Central Research Services, Charutar Arogya Mandal, Karamsad, Gujarat 388325, India
}

Correspondence should be addressed to Somashekhar M. Nimbalkar; somu_somu@yahoo.com

Received 17 June 2015; Accepted 28 July 2015

Academic Editor: Jean-Louis Excler

Copyright (C) 2015 Dipen V. Patel et al. This is an open access article distributed under the Creative Commons Attribution License, which permits unrestricted use, distribution, and reproduction in any medium, provided the original work is properly cited.

Appropriate feeding practices are the key contributor to reducing morbidities and mortalities in under-five children. A crosssectional questionnaire based survey of mothers of children aged less than 5years was conducted in 781 mothers. More than half of mothers $(57.5 \%)$ started feeding within an hour of birth, $55.9 \%$ gave exclusive breastfeeding for six months, $89.1 \%$ of the mothers stopped breastfeeding before two years of age, $18.2 \%$ of the mothers bottle-fed the babies, and $15.6 \%$ had problems during breastfeeding in first 6 months. Early initiation of breastfeeding within one hour of birth promoted exclusive breastfeeding, and breastfeeding for longer duration. Exclusive breastfeeding increased frequency of feeds. Multivariable logistic regression showed that initiation of breastfeeding after an hour of birth $(p=0.035)$, not providing exclusive breastfeeding for 6 months $(p<0.0001)$, unemployed mothers $(p=0.035)$, having two or more kids $(p=0.001)$, and complementary feeds given by person other than mother $(p=0.007)$ increased hospitalization. Starting breastfeeding after an hour of birth $(p=0.045)$, severe malnutrition $(p=0.018)$, and breastfeeding for $<$ two years $(p=0.026)$ increased rates of diarrhea. Breastfeeding practices were not optimum and interventions to improve these practices need to be strengthened.

\section{Introduction}

Appropriate Infant and Young Child Feeding (IYCF) practices are essential for optimal growth, cognitive development, and overall well-being in early vulnerable years of life. Malnutrition contributes to about $60 \%$ of under-five mortality worldwide annually and over two-thirds of these are due to inappropriate feeding practices [1]. An analysis showed that appropriate breastfeeding and complementary feeding practices can alone prevent under-five deaths by $19 \%$ [2].

The World Health Organization (WHO) recommends exclusive breastfeeding for the first six months of life and the addition of complementary feeds from six months onwards, with continued breastfeeds till at least two years of age $[3,4]$.

Apart from exclusive breastfeeding initially, time of introduction, content, and consistency of complementary feeds are critical for early nutrition. The early introduction of complementary feeds before the age of six months can lead to displacement of breast milk and increased risk of infections, besides the babies being physiologically immature. Similarly, inadequate and inappropriate complementary feeding with unhygienic practices leads to recurrent and persistent infections and malnutrition which is followed by growth retardation, immunodeficiency, and eventually fatal outcomes. This is a concern for Indian scenario, where previous studies have suggested inability to maintain exclusive breastfeeding and late introduction of complementary feeds [5-7]. Evidence based studies have stressed the importance of human milk and concluded that infant feeding should be considered as basic health issue rather than lifestyle choice [8].

Breastfeeding though is a natural act; it is a behavior that needs to be learned. Mothers and other caregivers need active assistance for optimum breastfeeding practices. The Global Strategy for Infant and Young Child Feeding describes the essential interventions to promote, protect, and support exclusive breastfeeding [9]. 
Multiple efforts are being envisaged to improve feeding practices in children. WHO and United Nations Children's Fund (UNICEF) launched the Baby-Friendly Hospital Initiative in 1992, Integrated Management of Childhood Illness (IMCI) in mid-1990s, and IYCF in 2002 which stress the importance of breastfeeding. All these programs have been adopted by India since the last one to two decades to promote appropriate feeding practices in children under-five years.

Appropriate breastfeeding and complementary feeding practices depend on accurate information and support from the family, community, and healthcare system. Inadequate knowledge about feeding practices is an equally important determinant of malnutrition, as is the lack of adequate and hygienic food. However, in spite of all the efforts deployed as information, education, or training campaigns, the prevalence of proper feeding practices remains low [10].

India hosts more than one-third of the world's children who are wasted. From under-five children in India, $43 \%$ are underweight and $48 \%$ are stunted due to chronic undernutrition. Only $25 \%$ of newborns were put to the breast within one hour of birth and $46 \%$ are exclusively breastfed [10].

Multiple factors are responsible for this slow improvement in nutritional health indicators in India. According to World Bank report, India's Integrated Child Development Services (ICDS) needs to undergo significant changes to address the current malnutrition crisis in India [11].

As there is a paucity of literature on the feeding practices in this region, the present study was undertaken to assess the feeding practices of mothers having children under-five years of age. The interaction of sociodemographic variables with feeding practices and associations of childhood morbidity were studied.

\section{Materials and Methods}

2.1. Study Design. This study is designed as a questionnaire based survey.

2.2. Study Site and Population. This study was conducted at the pediatrics outpatient department (OPD) catering to children from birth to 18 years of age attached to a tertiary care Shree Krishna Hospital (SKH) of Pramukhswami Medical College located at Karamsad from Anand district of Gujarat, India, over a period of four months from October 2013 to January 2014. The hospital caters to Anand and Kheda districts of Gujarat. Mothers having children less than five years of age attending the clinic for immunizations, growth monitoring, treatment of minor illnesses, health check-ups, follow-up, and so forth were interviewed after taking an informed consent. All children with known nonnutritional congenital or acquired reasons (e.g., congenital heart diseases, cerebral palsy, genetic disorders, and tuberculosis) of failure to thrive were excluded.

2.3. Study Procedure. The questionnaire consisted of 39 questions on sociodemographic variables, feeding practices, and morbidities of children (hospitalization, diarrhea, and acute respiratory illnesses). Weight of the child was taken on a digital weighing scale of Zeal Medical Pvt. Ltd. Weight for age classification was used to estimate the nutritional status of children using the $z$-score cut-off point of WHO Global Database on Child Growth and Malnutrition [12]. Nutritional status was defined with $z$-score of $<-3 z$ as severe undernutrition, between -3 and -2 as moderate undernutrition, between -2 and $+2 z$ as normal, and $>+2$ as overweight. The questionnaire was in English but the interviews were conducted in local language (Gujarati/Hindi/English) as per mothers' convenience. The OPD runs between 9:00 a.m. and 1:30 p.m. and between 3:00 p.m. and 5:00 p.m. Interviews were conducted between 10 a.m. and 1:00 p.m. and each interview took approximate 15 to 25 minutes to finish. Data was entered on a predesigned, structured questionnaire on Magpie (Datadyne) application on a Generation 2 iPAD running on iOS6 and later uploaded on the server website on daily basis. The services provided by the website were free of charge. Data was transcribed to Microsoft Office Excel 2010 and then imported to SPSS 14 (SPSS Inc., Chicago, IL, USA) for analysis after validation and cleaning.

2.4. Statistical Analysis. Descriptive statistics were used to depict the sociodemographic characteristics, clinical profile, and feeding practices of the study population. Associations between feeding practices and sociodemographic variables as well as between feeding practices, sociodemographic variables, and hospital admissions at univariate level were determined using chi-square test. Multivariable logistic regression was performed to determine independent associations. Odds ratio $(\mathrm{OR})$ was calculated and significance was considered at $p$ value $<0.05$.

2.5. Ethical Considerations. The study was approved by human research ethics committee of HM Patel Centre for Medical Care and Education, Karamsad.

\section{Results}

Out of 6385 children who visited pediatrics OPD between October 2013 and January 2014, 4416 (69.16\%) were below 5 years of age. As the interviews were conducted between 10:00 $\mathrm{h}$ and 13:00 h, a total of 910 children could be approached. Out of these, 811 were accompanied by their mothers. Twenty-four mothers did not give consent due to time constraints while 6 were not eligible because of the known cause of failure to thrive (i.e., 3 had congenital heart diseases, 2 had cerebral palsy, and 1 had pulmonary tuberculosis). Thus 781 (97\%) eligible mothers participated in the study.

The mean (SD) age of participating mothers was 27.18 (3.77) years (range: 19 to 42 ). Majority of mothers $(86.1 \%$ ) had studied up to 10 th standard. Very few $(2.6 \%)$ mothers had multifetal pregnancy. The monthly family income was quite skewed ranging from Rs. 2000 to Rs. 45000. Majority of the children belong to age group of six months to two years $(54.8 \%)$ and had an older sibling (55.5\%). The mean (SD) birth weight of the children was 2708.94 (510.92) grams (range: 900 to 4500). Most of them suffered from diarrhea $(61.7 \%)$ and cough and cold (77.6\%) and required hospitalization (41.9\%) (Table 1). 
TABLE 1: Sociodemographic variables.

\begin{tabular}{|c|c|}
\hline Sociodemographic variable & Frequency \\
\hline Age (years) of the mothers, mean (SD) & 27.18 (3.77), range: 19 to 42 years \\
\hline Mother's education less than 4 th standard & 109 of $780(13.97 \%)$ \\
\hline Low birth weight children & 195 of $768(25.4 \%)$ \\
\hline Monthly family income less than Rs. 15000 & 570 of $765(74.5 \%)$ \\
\hline Multiparous mothers & 431 of $777(55.5 \%)$ \\
\hline Number of children who required hospitalization & 327 of $781(41.9 \%)$ \\
\hline Number of children who had diarrhea & 482 of $781(61.7 \%)$ \\
\hline Number of children who had cough-cold & 606 of $780(77.6 \%)$ \\
\hline Multifetal pregnancy & 17 of $778(2.2 \%)$ \\
\hline Number of mothers who had problems during breastfeeding during first 6 months after delivery & 120 of $771(15.6 \%)$ \\
\hline Family support for breastfeeding & 76 of $766(10 \%)$ \\
\hline Male gender & 476 of $780(61 \%)$ \\
\hline Bottle feeding & 142 of $771(18.4 \%)$ \\
\hline Employed women & 138 of $779(17.7 \%)$ \\
\hline Hindu religion & 653 of $779(83.8 \%)$ \\
\hline Started breastfeeding within 1st hour of delivery & 443 of $771(57.5 \%)$ \\
\hline \multicolumn{2}{|l|}{ Age of the child (of total 767) } \\
\hline Less than 6 months & $60(7.8 \%)$ \\
\hline 6 months to 24 months & $420(54.8 \%)$ \\
\hline More than 24 months & $287(37.4 \%)$ \\
\hline Use of soap to wash hands for children more than 6 months before feeding & 404 of $687(58.8 \%)$ \\
\hline Washing utensils after each feed & 532 of $697(76.3 \%)$ \\
\hline Washing hand of mother before each feed for children more than 6 months age & 499 of $692(72.1 \%)$ \\
\hline \multicolumn{2}{|l|}{ Who feeds the child more than 6 months } \\
\hline Mother & $600(86.3 \%)$ \\
\hline Other person & $95(13.7 \%)$ \\
\hline Exclusive breastfeeding for 6 months in children more than 2 years & 160 of $286(55.9 \%)$ \\
\hline Breastfeeding stopped less than 2 years of age in children more than 2 years & 253 of $284(89.1 \%)$ \\
\hline
\end{tabular}

As per WHO definition of nutritional status as per weight for age [12], 411 (55.5\%) were normal, 78 (10.5\%) had moderate undernutrition, 149 (20.1\%) were severely malnourished, and $102(13.8 \%)$ were overweight. Total duration of breastfeeding more than 1 year $(p=0.002)$ and exclusive breastfeeding for 6 months were associated with lower rates of severe malnutrition $(p=0.003)$.

More than half of the mothers (57.5\%) started breastfeeding within one hour of child's birth, gave exclusive breastfeeding for recommended 6 months (55.9\%), and continued to breastfeed in the second year of life (50.7\%). Most mothers did not face any problem during breastfeeding in the first six months (84.4\%) and did not bottle-feed the baby (81.6\%). More than half of the mothers washed their hands $(72.1 \%)$ before giving feeds and washed utensils after each feed (76.3\%). Only $26.5 \%$ of the mothers who gave complementary feeds stored the food in oven or refrigerator and $42.2 \%$ always rewarmed the stored food before giving; however, $61.9 \%$ of the mothers often gave freshly prepared food. The complementary feed was given mostly by the mother $(86.6 \%)$ (Table 1).
Primiparous mothers and mothers with multifetal pregnancy started breastfeeding early. Mothers who breastfed their children within 1st hour of birth were more likely to give exclusive breastfeeding for 6 months, breastfeed for a longer duration, and engage in bottle-feeding the baby. Maternal education was not associated with time of starting breastfeeding. Mothers who exclusively breastfed their children for recommended period of 6 months gave breastfeeding for longer duration and frequently breastfed their child in a day (Tables 2 and 3).

Multivariable logistic model revealed that initiation of breastfeeding within one hour, exclusive breastfeeding for six months, maternal employment, primipara status, mother feeding the child, and overweight significantly reduced hospital admissions. The predictive value of the model was good with $65.3 \%$ correct classification rate (Table 4 ).

Severe malnutrition, not starting breastfeeding within 1st hour of birth, and stopping breastfeeding before 2 years were associated with increased diarrhea episodes. The predictive value of the model was good with $67.5 \%$ correct classification rate (Table 5). 
TABLE 2: Univariate analysis for time of starting breastfeeding after birth in children more than 2 years.

\begin{tabular}{|c|c|c|c|}
\hline \multirow[b]{2}{*}{ Variables } & \multicolumn{2}{|c|}{ When did breastfeeding start? } & \multirow[b]{2}{*}{$p$ value } \\
\hline & $\begin{array}{l}\text { After 1st hour of birth } \\
\qquad N(\%)\end{array}$ & $\begin{array}{l}\text { Within 1st hour of birth } \\
\qquad N(\%)\end{array}$ & \\
\hline \multicolumn{4}{|l|}{ Elder siblings } \\
\hline No & $129(37.7)$ & $213(62.3)$ & \multirow{2}{*}{0.018} \\
\hline More than/equal to 1 & $197(46.2)$ & $229(53.8)$ & \\
\hline \multicolumn{4}{|l|}{ Births } \\
\hline Multifetal & $2(12.5)$ & $14(87.5)$ & \multirow{2}{*}{0.014} \\
\hline Single & $325(43.2)$ & $428(56.8)$ & \\
\hline \multicolumn{4}{|l|}{ Studied up to } \\
\hline Less than 4th standard & $49(45.4)$ & $59(54.6)$ & \multirow{2}{*}{0.51} \\
\hline 4th standard and above & $278(42)$ & $384(58)$ & \\
\hline \multicolumn{4}{|l|}{ Bottle feeding* } \\
\hline No & $283(86.8)$ & $346(79.0)$ & \multirow{2}{*}{0.003} \\
\hline Yes & $43(13.2)$ & $92(21.0)$ & \\
\hline \multicolumn{4}{|l|}{ Duration of breastfeeding* } \\
\hline Less than 2 years & $293(97.1)$ & $371(93.5)$ & \multirow{2}{*}{0.032} \\
\hline 2 years or more & $9(2.9)$ & $26(6.5)$ & \\
\hline \multicolumn{4}{|l|}{ Exclusive breastfeeding ${ }^{*}$} \\
\hline Other than 6 months/more than 1 year & $161(49.1)$ & $171(38.8)$ & \multirow{2}{*}{0.004} \\
\hline 6 months & $167(50.9)$ & $270(61.2)$ & \\
\hline
\end{tabular}

${ }^{*}$ Column percentages are reported.

Children of unemployed mothers and having severe malnutrition were more likely to encounter cough/cold episodes. Hygiene of hands and utensils showed inverse association with cough/cold episodes. The predictive value of the model was good with $79.5 \%$ correct classification rate (Table 5).

\section{Discussion}

Early initiation of breastfeeding and exclusive breastfeeding of children below six months are considered the most decisive indicators for assessing breastfeeding practices [13].

In the present study $57.5 \%$ of the children received breastfeeding within one hour of birth. Similarly a study done at Allahabad showed $55.8 \%$ of the mothers initiating breastfeeding within six hours [14]. However, the National Family Health Survey (NFHS-3) 2005-2006 estimates are lower for India and Gujarat being $23.5 \%$ and $25.4 \%$, respectively [15]. Another recent Indian study from Madhya Pradesh recorded similar low $26 \%$ mothers initiating breastfeeding in first hour [16]. The higher percentage found in our study can be attributed to the fact that the NFHS was done about 9 years ago and was based on community based survey. Discrepancies from other Indian studies can be due to regional and cultural differences. Delayed initiation of breastfeeding has been recognized as a risk factor for neonatal mortality and according to a previous study, an estimated $22 \%$ of neonatal deaths could be prevented if breastfeeding was started within the first hour after birth [17]. Even though there is an increase compared to the NFHS data, the current rate is still inadequate to achieve the estimated mortality benefits of early initiation.

Exclusive breastfeeding during the first 6 months and therefore timely introduction of complementary feeding have many proven advantages to both the mother and the child and are therefore the prime focus in infant feeding promotional activities. In our study we observed $55.9 \%$ of the infants exclusively breastfeed till complete six months. Similar rates were observed by Das et al. (58.7\%) and Banapurmath et al. $(60 \%)[18,19]$. Indian studies by Kulkarni et al. $(70.2 \%)$ and Nimbalkar et al. (85.6\%) recorded much higher rates $[20,21]$. Last NFHS-3 data of India and Gujarat shows $46.3 \%$ and $47.8 \%$ of infants in $0-5$ months of age exclusively breastfed. And from the 6th month onwards $55.8 \%$ and $57.7 \%$ of infants received complementary feeding in India and Gujarat, respectively. Conversely, other Indian studies from Delhi showed lower recordings with $16.5 \%$ and $17.5 \%$ mothers starting timely complementary feeding $[5,6]$. Appropriate duration of exclusive breastfeeding with timely introduction of complementary feeds is essential for growth and well-being of the child. An estimated $10 \%$ to $15 \%$ of under-five deaths in resource poor countries could be prevented if $90 \%$ of babies were exclusively breastfed for the first 6 months [2]. On multivariate analysis we found that children not exclusively breastfed for 6 months and started on breastfeeding after one hour of birth had significantly higher hospital admissions.

The NFHS-3 data for Gujarat state reports $93.5 \%$ of mothers breastfeeding between 6 and 9 months, which is similar to our data of $93.3 \%$ after 6 months. However, in 
TABLE 3: Univariate analysis for exclusive breastfeeding in children more than 2 years.

\begin{tabular}{|c|c|c|c|}
\hline \multirow[b]{2}{*}{ Variables } & \multicolumn{2}{|c|}{ Duration of exclusive breastfeeding } & \multirow[b]{2}{*}{$p$ value } \\
\hline & $\begin{array}{l}\text { Other than } 6 \text { months } \\
\qquad N(\%)\end{array}$ & $\begin{array}{l}6 \text { months } \\
N(\%)\end{array}$ & \\
\hline \multicolumn{4}{|c|}{ Problems in breastfeeding in first 6 months } \\
\hline No & $101(42.1)$ & $139(57.9)$ & \multirow{2}{*}{0.095} \\
\hline Yes & $25(55.6)$ & $20(44.4)$ & \\
\hline \multicolumn{4}{|l|}{ Studied up to } \\
\hline Less than 4 th standard & $18(46.2)$ & $21(53.8)$ & \multirow{2}{*}{0.776} \\
\hline 4th standard and above & $108(43.7)$ & $139(56.3)$ & \\
\hline \multicolumn{4}{|l|}{ Bottle-feeding } \\
\hline No & $96(42.9)$ & $128(57.1)$ & \multirow{2}{*}{0.438} \\
\hline Yes & $30(48.4)$ & $32(51.6)$ & \\
\hline \multicolumn{4}{|l|}{ Occupation } \\
\hline Employed & $22(47.8)$ & $24(52.2)$ & \multirow{2}{*}{0.574} \\
\hline Housewife & $104(43.3)$ & $136(56.7)$ & \\
\hline \multicolumn{4}{|l|}{ Sex of child } \\
\hline Female & $56(50.5)$ & $55(49.5)$ & \multirow{2}{*}{0.083} \\
\hline Male & $70(40.0)$ & $105(60.0)$ & \\
\hline \multicolumn{4}{|l|}{ Religion } \\
\hline Christian & $7(50.0)$ & $7(50.0)$ & \multirow{3}{*}{0.498} \\
\hline Hindu & $111(44.9)$ & $136(55.1)$ & \\
\hline Muslin & $8(33.3)$ & $16(66.7)$ & \\
\hline \multicolumn{4}{|l|}{ Nutrition } \\
\hline Normal & $65(40.4)$ & $96(59.6)$ & \multirow{4}{*}{0.581} \\
\hline Moderate malnutrition & $21(45.7)$ & $25(54.3)$ & \\
\hline Overweight & $1(50.0)$ & $1(50.0)$ & \\
\hline Severe malnutrition & $35(50.0)$ & $35(50.0)$ & \\
\hline \multicolumn{4}{|l|}{ Duration of breastfeeding* } \\
\hline Less than 6 Months & $47(14.7)$ & $0(0.0)$ & \multirow{3}{*}{$<0.0001$} \\
\hline 6 to 23 months & $260(81.2)$ & $361(94.2)$ & \\
\hline 24 months or more & $13(4.1)$ & $22(5.8)$ & \\
\hline \multicolumn{4}{|c|}{ Number of breastfeeds per 24 hours* } \\
\hline Less than 8 times & $270(80.4)$ & $312(71.5)$ & \multirow{2}{*}{0.005} \\
\hline 8 times or more & $66(19.6)$ & $124(28.5)$ & \\
\hline
\end{tabular}

${ }^{*}$ Column percentages are reported.

the second year the breastfeeding rate observed in our study (50.7\%) was lower than seen in NFHS-3 data (76.8\%).

The rate of bottle-feeding was $18.4 \%$ similar to what was observed in NFHS-3 and in other studies. It is one of the optional indicators of IYCF practices. Bottle-feeding should be discouraged-bottles are difficult to clean and sterilize and are the source of infection, and artificial teats cause "nipple confusion" thus interfering with breastfeeding. The bottlefeeding was $11 \%$ in a study from urban population [22]. We found higher rates of bottle-feeding in children who were breastfed within 1st hour of birth.

Maternal illiteracy has been associated with suboptimal feeding practices [23]. We did not observe associations between maternal education with duration and initiation of breastfeeding on univariate analysis. Maternal education plays a huge role in increasing the receptivity of mothers towards correct practices. Studies from India have suggested significant association of maternal literacy and timely initiation of complementary feeding $[5,24,25]$. Lower literacy in mothers, in addition to lack of knowledge about correct practices and recommendations, makes routine counseling by community health workers also ineffective [26].

We did not find significant association of maternal occupation with initiation of breastfeeding and duration of exclusive breastfeeding despite housewives supposedly having more time available to feed their infants. One of the probable reasons for this is that working mothers carry their children at workplace and they are able to provide breastfeeding. A study in Malaysia reported that facility at workplace allowing mothers a flexible time to express 
TABLE 4: Variables associated with hospital admissions in children aged more than 6 months.

\begin{tabular}{|c|c|c|c|c|}
\hline \multirow{2}{*}{ Variables } & \multirow{2}{*}{$p$ value } & \multirow{2}{*}{ OR } & \multicolumn{2}{|c|}{$95 \% \mathrm{CI}$ for OR } \\
\hline & & & Lower & Upper \\
\hline \multicolumn{5}{|l|}{ Starting of breastfeeding } \\
\hline After an hour of birth & 0.035 & 1.482 & 1.027 & 2.138 \\
\hline \multicolumn{5}{|l|}{ Within hour (reference category) } \\
\hline \multicolumn{5}{|l|}{ Exclusive breastfeeding } \\
\hline \multicolumn{5}{|l|}{6 months (reference category) } \\
\hline Less than 6 months or one year and more than 1 year & $<0.0001$ & 2.100 & 1.450 & 3.039 \\
\hline \multicolumn{5}{|l|}{ Occupation } \\
\hline Employed & 0.035 & 0.585 & 0.356 & 0.963 \\
\hline \multicolumn{5}{|l|}{ Housewife (reference category) } \\
\hline \multicolumn{5}{|l|}{ Elder siblings } \\
\hline One and more & 0.001 & 1.923 & 1.326 & 2.788 \\
\hline \multicolumn{5}{|l|}{ No (reference category) } \\
\hline \multicolumn{5}{|l|}{ Feeding given by } \\
\hline \multicolumn{5}{|l|}{ Mostly mother (reference category) } \\
\hline Other & 0.007 & 2.070 & 1.219 & 3.515 \\
\hline Nutritional status of children & 0.006 & & & \\
\hline Moderate malnutrition & 0.939 & 1.024 & 0.562 & 1.866 \\
\hline Overweight & 0.011 & 0.441 & 0.233 & 0.831 \\
\hline Severe malnutrition & 0.052 & 1.578 & 0.996 & 2.499 \\
\hline Normal (reference category) & & & & \\
\hline
\end{tabular}

breast milk helps in maintaining lactation [27]. Conversely, there have been other studies observing maternal occupation associated with suboptimal feeding practices [28-31]. On multivariate analysis, we found higher hospitalizations for children of housewives.

In our study we found an association of multifetal births with initiation of breastfeeding within one hour of birth and no relation with duration of exclusive breastfeeding. However, small sample size in multiple pregnancies $(N=16)$ was a limitation. Studies from Japan recorded significantly lower rate of exclusive breastfeeding and shorter duration of breastfeeding among twins or triplets than among singleton babies [32, 33]. Multiple births are seen as a hindrance to breastfeeding because of frequent association of prematurity with multiple pregnancies with lack/weakness of sucking reflex [34]. However in a study done in Canada on term newborns (singleton and twin), mothers of twins were again found less likely to exclusively breastfeed (adjusted odds ratio (OR) 0.30, 95\% confidence interval (CI) 0.25-0.36) [35]. Prenatal education and counselling regarding feeding practices, such as alternating breasts, increases the initiation and duration of breastfeeding $[3,36]$. Supporting and educating the families with birth of multiple babies increased breastfeeding by $74 \%$ [34].

In our study primiparous mothers initiated breastfeeding earlier than multiparous mothers. Microbial contamination of feeds is a major cause of infections leading to higher hospital admissions. A study from Madhya Pradesh showed significant association of hygienic practices and malnutrition in infants [16]. Meager data on hygiene practices during food preparation and feeding are available. Results of a study in north western Nigeria showed that only $28 \%$ of mothers washed their hands before preparing foods [37]. A study from slums of Dhaka showed 33\% of the mothers exclusively breastfeeding while $54 \%$ of the nonexclusively breastfeeding mothers did not clean their child's hands before feeding [38]. Similarly another study from India showed just $17 \%$ hand cleaning before feeding [24]. We found higher values on hygienic practices like hand washing of mothers and washing utensils after each feed, but it was not up to the mark. On multivariate analysis we found reduced episodes of cough and cold in children whose mothers washed their hands and utensils sometimes as against the reference category of always washing hands and utensils, respectively. These findings may be because of varied culture, hygienic habits, and home environment, which were not evaluated in detail in the current study.

We found an association between hospital admissions of children with feeding given by person other than mother. Study from Maharashtra showed feeding given by foster mothers (elder brother/sister) as a major determinant of faulty feeding practices [39]. Mothers having two or more kids and severe malnutrition in children were associated with higher rates of hospital admission while overweight was actually protecting against hospital admissions.

We conclude that there has been slight improvement in feeding practices since the last NFHS survey in 2005-2006. However, the current rates of proper feeding practices are still well below the guidelines for Infant and Young Child Feeding. Late initiation of breastfeeding, nonexclusive breastfeeding, bottle-feeding, maternal illiteracy, and unhygienic feeding practices are still prevailing. Though more emphasis in terms 
TABLE 5: Variables associated with diarrhea and cough/cold episodes in children age more than 6 months.

\begin{tabular}{|c|c|c|c|c|}
\hline \multirow{2}{*}{ Variables } & \multirow{2}{*}{$p$ value } & \multirow{2}{*}{ OR } & \multicolumn{2}{|c|}{$95 \%$ CI for OR } \\
\hline & & & Lower & Upper \\
\hline \multicolumn{5}{|c|}{ Variables associated with diarrhea in children aged more than 6 months } \\
\hline \multicolumn{5}{|l|}{ Starting of breastfeeding } \\
\hline After an hour of birth & 0.045 & 1.479 & 1.009 & 2.166 \\
\hline \multicolumn{5}{|c|}{ Within hour (reference category) } \\
\hline Nutritional status of children & 0.029 & & & \\
\hline Moderate malnutrition & 0.758 & 0.909 & 0.497 & 1.666 \\
\hline Overweight & 0.209 & 0.695 & 0.394 & 1.227 \\
\hline Severe malnutrition & 0.018 & 1.853 & 1.111 & 3.089 \\
\hline \multicolumn{5}{|l|}{ Normal (reference category) } \\
\hline \multicolumn{5}{|l|}{ Duration of breastfeeding } \\
\hline Up to 2 years & 0.026 & 2.506 & 1.114 & 5.638 \\
\hline \multicolumn{5}{|c|}{2 years or more (reference category) } \\
\hline \multicolumn{5}{|c|}{ Variables associated with cough and cold in children aged more than 6 months } \\
\hline \multirow{2}{*}{\multicolumn{5}{|c|}{$\begin{array}{l}\text { Hand washing before each feed } \\
\text { Always (reference category) }\end{array}$}} \\
\hline & & & & \\
\hline Sometimes & 0.039 & 0.620 & 0.394 & 0.977 \\
\hline Nutritional status of child & 0.115 & & & \\
\hline Moderate malnutrition & 0.590 & 1.221 & 0.590 & 2.527 \\
\hline Overweight & 0.636 & 1.182 & 0.591 & 2.363 \\
\hline Severe malnutrition & 0.015 & 2.135 & 1.159 & 3.933 \\
\hline \multicolumn{5}{|l|}{ Normal (reference category) } \\
\hline \multicolumn{5}{|c|}{ Washing utensils after each feed } \\
\hline \multicolumn{5}{|c|}{ After each feed (reference category) } \\
\hline Sometimes & 0.001 & 0.457 & 0.282 & 0.740 \\
\hline \multicolumn{5}{|l|}{ Occupation } \\
\hline Employed & 0.022 & 0.546 & 0.325 & 0.916 \\
\hline Housewife (reference categ & & & & \\
\hline
\end{tabular}

of antenatal counseling, hospital delivery, and training of grass root workers on IYCF is being conducted to improve child feeding practices, they are still inadequate to improve short term and long term outcomes of childhood nutrition. There is an alarming need of a prospective educational intervention study or new innovative practices to achieve the optimum feeding practices in children under the age of five years.

\section{Conflict of Interests}

The authors declare that there is no conflict of interests regarding the publication of this paper.

\section{Acknowledgment}

The authors acknowledge Ms. Amee Amin for English language check of the paper.

\section{References}

[1] Ministry of Women and Child Development FaNB and Government of India, National Guidelines on Infant and Young Child Feeding, Ministry of Women and Child Development FaNB, Government of India, New Delhi, India, 2nd edition, 2006.

[2] G. Jones, R. W. Steketee, R. E. Black, Z. A. Bhutta, and S. S. Morris, "How many child deaths can we prevent this year?" The Lancet, vol. 362, no. 9377, pp. 65-71, 2003.

[3] WHO, Global Strategy on Infant and Young Child Feeding, World Health Organzation, Geneva, Switzerland, 2003.

[4] World Health Organization, Complementary Feeding: Report of the Global Consultation. Summary of Guiding Principles for Complementary Feeding of the Breastfed Child, World Health Organization, Geneva, Switzerland, 2003.

[5] A. Aggarwal, S. Verma, and M. M. A. Faridi, "Complementary feeding-reasons for inappropriateness in timing, quantity and consistency," Indian Journal of Pediatrics, vol. 75, no. 1, pp. 49$53,2008$. 
[6] V. Sethi, S. Kashyap, and V. Seth, "Effect of nutrition education of mothers on infant feeding practices," The Indian Journal of Pediatrics, vol. 70, no. 6, pp. 463-466, 2003.

[7] P. L. Engle, "Infant feeding styles: barriers and opportunities for good nutrition in India," Nutrition Reviews, vol. 60, supplement 5, pp. S109-S114, 2002.

[8] American Academy of Pediatrics Section on Breastfeeding, "Breastfeeding and the use of human milk," Pediatrics, vol. 129, no. 3, pp. e827-e841, 2012.

[9] Organization WH. WHO, "Exclusive breastfeeding," 2015, http://www.who.int/nutrition/topics/exclusive_breastfeeding/ en/.

[10] Unicef India, Nutrition-Latest Stories, Unicef India, 2014, http://www.unicef.org/india/nutrition.html.

[11] World Bank, Urgent Action Needed to Overcome Persistent Malnutrition in India, World Bank, New Delhi, India, 2006, http://go.worldbank.org/N3YWAHV3I0.

[12] World Health Organization, Global Database on Child Growth and Malnutrition, World Health Organization, Geneva, Switzerland, 2013, http://www.who.int/nutgrowthdb/about/introduction/ en/index $5 . h$ tml.

[13] World Health Organization, Indicators for Assessing Infant and Young Child Feeding Practices: Part 1 Definitions, WHO, Geneva, Switzerland, 2008.

[14] D. Kumar, N. K. Goel, P. C. Mittal, and P. Misra, "Influence of infant-feeding practices on nutritional status of under-five children," Indian Journal of Pediatrics, vol. 73, no. 5, pp. 417-421, 2006.

[15] F. Arnold, S. Parasuraman, P. Arokiasamy, and M. Kothari, Nutrition in India, International Institute for Population Sciences, Mumbai, India, 2005.

[16] I. I. Meshram, M. R. Kodavanti, G. R. Chitty, and et al, "Influence of feeding practices and associated factors on the nutritional status of infants in rural areas of Madhya Pradesh state, India," Asia-Pacific Journal of Public Health, vol. 27, no. 2, pp. NP1345-NP1361, 2015.

[17] K. M. Edmond, C. Zandoh, M. A. Quigley, S. Amenga-Etego, S. Owusu-Agyei, and B. R. Kirkwood, "Delayed breastfeeding initiation increases risk of neonatal mortality," Pediatrics, vol. 117, no. 3, pp. e380-e386, 2006.

[18] C. R. Banapurmath, M. C. Nagaraj, S. Banapurmath, and N. Kesaree, "Breastfeeding practices in villages of Central Karnataka," Indian Pediatrics, vol. 33, no. 6, pp. 477-479, 1996.

[19] N. Das, D. Chattopadhyay, S. Chakraborty, and A. Dasgupta, "Infant and young child feeding perceptions and practices among mothers in a rural area of West Bengal, India," Annals of Medical and Health Sciences Research, vol. 3, no. 3, pp. 370375, 2013.

[20] R. N. Kulkarni, S. Anjenya, and R. Gujar, "Breast feeding practices in an urban community of Kalamboli, Navi Mumbai," Indian Journal of Community Medicine, vol. 29, no. 4, pp. 179180, 2004.

[21] A. S. Nimbalkar, V. V. Shukla, A. G. Phatak, and S. M. Nimbalkar, "Newborn care practices and health seeking behavior in urban slums and villages of Anand, Gujarat," Indian Pediatrics, vol. 50, no. 4, pp. 408-410, 2013.

[22] N. A. Bhosale, S. G. Deshpande, S. P. Zodpey, S. N. Jog, and N. D. Vasudeo, "Infant feeding practices in urban population-a clinic based study," Indian Journal of Medical Sciences, vol. 51, no. 10, pp. 396-399, 1997.
[23] U. Lawan, A. Sani, G. Amole, and M. Jahum, "Age-appropriate feeding practices and nutritional status of infants attending child welfare clinic at a Teaching Hospital in Nigeria," Journal of Family and Community Medicine, vol. 21, no. 1, pp. 6-12, 2014.

[24] M. Chauhan, R. Bala, D. Nandan, and S. K. Misra, "Complementary feeding practices in rural area of district Agra," Indian Journal of Public Health, vol. 51, no. 1, pp. 66-67, 2007.

[25] S. Rao, "Study of complementary feeding practices among mothers of children aged six months to two years-a study from coastal south India," Australasian Medical Journal, vol. 4, no. 5, pp. 252-257, 2011.

[26] A. Rahman, Z. Haq, S. Sikander, I. Ahmad, M. Ahmad, and A. Hafeez, "Using cognitive-behavioural techniques to improve exclusive breastfeeding in a low-literacy disadvantaged population," Maternal and Child Nutrition, vol. 8, no. 1, pp. 57-71, 2012.

[27] R. Amin, Z. Said, R. Sutan, S. Shah, A. Darus, and K. Shamsuddin, "Work related determinants of breastfeeding discontinuation among employed mothers in Malaysia," International Breastfeeding Journal, vol. 6, no. 1, article 4, 2011.

[28] B. Al-Sahab, A. Lanes, M. Feldman, and H. Tamim, "Prevalence and predictors of 6-month exclusive breastfeeding among Canadian women: a national survey," BMC Pediatrics, vol. 10, article 20, 2010.

[29] A. H. El-Gilany, E. Shady, and R. Helal, "Exclusive breastfeeding in Al-Hassa, Saudi Arabia," Breastfeeding Medicine, vol. 6, no. 4, pp. 209-213, 2011.

[30] A. M. Seid, M. E. Yesuf, and D. N. Koye, "Prevalence of Exclusive Breastfeeding Practices and associated factors among mothers in Bahir Dar city, Northwest Ethiopia: a community based cross-sectional study," International Breastfeeding Journal, vol. 8, no. 1, article 14, 2013.

[31] K. L. Tan, "Factors associated with exclusive breastfeeding among infants under six months of age in peninsular Malaysia," International Breastfeeding Journal, vol. 6, no. 1, article 2, 2011.

[32] Y. Yokoyama and S. Ooki, "Breast-feeding and bottle-feeding of twins, triplets and higher order multiple births," Japanese Journal of Public Health, vol. 51, no. 11, pp. 969-974, 2004.

[33] Y. Yokoyama, S. Wada, M. Sugimoto, M. Katayama, M. Saito, and J. Sono, "Breastfeeding rates among singletons, twins and triplets in Japan: a population-based study," Twin Research and Human Genetics, vol. 9, no. 2, pp. 298-302, 2006.

[34] E. G. Damato, D. A. Dowling, E. A. Madigan, and C. Thanattherakul, "Duration of breastfeeding for mothers of twins," Journal of Obstetric, Gynecologic, \& Neonatal Nursing, vol. 34, no. 2, pp. 201-209, 2005.

[35] S. D. McDonald, E. Pullenayegum, B. Chapman et al., "Prevalence and predictors of exclusive breastfeeding at hospital discharge," Obstetrics \& Gynecology, vol. 119, no. 6, pp. 1171-1179, 2012.

[36] K. K. Gromada and A. K. Spangler, "Breastfeeding twins and higher-order multiples," Journal of Obstetric, Gynecologic, \& Neonatal Nursing, vol. 27, no. 4, pp. 441-449, 1998.

[37] A. K. S. Matthew, A. D. Amodu, I. Sani, and S. D. Solomon, "Infant feeding practices and nutritional status of children in north western Nigeria," Asian Journal of Clinical Nutrition, vol. 1, no. 1, pp. 12-22, 2009.

[38] F. Saleh, F. Ara, M. A. Hoque, and M. S. Alam, "Complementary feeding practices among mothers in selected slums of Dhaka city: a descriptive study," Journal of Health, Population and Nutrition, vol. 32, no. 1, Article ID 24847597, pp. 89-96, 2014. 
[39] P. A. Somaiya and R. V. Awate, "Infant feeding practices in the urban slum of Karad in west Maharashtra., Journal of the Indian Medical Association, vol. 88, no. 1, pp. 13-15, 1990. 


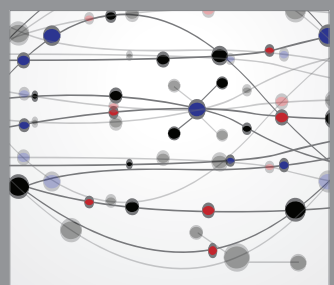

The Scientific World Journal
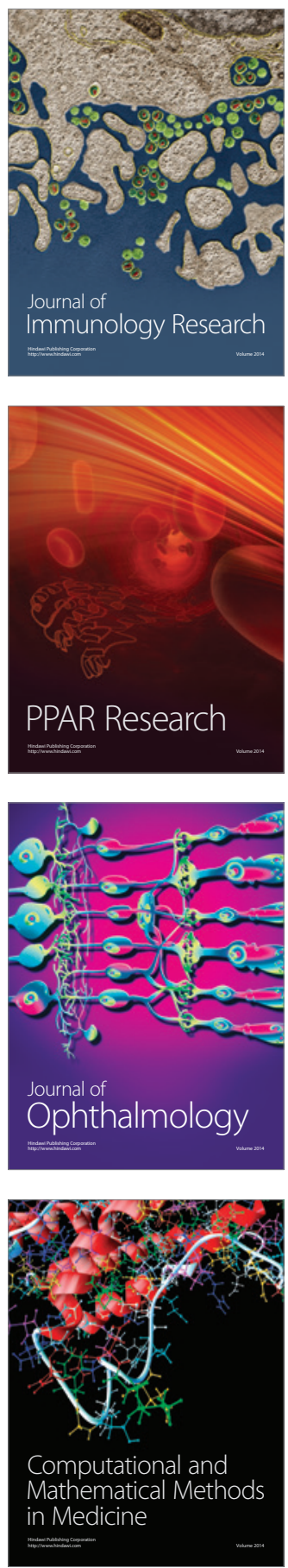

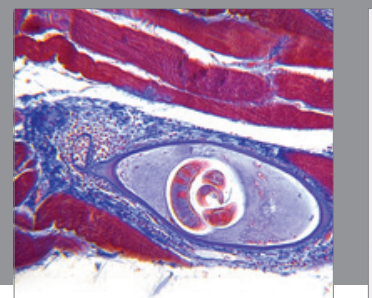

Gastroenterology

Research and Practice
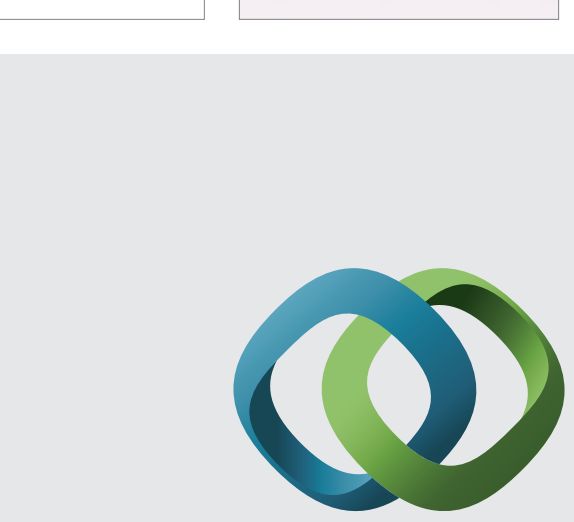

\section{Hindawi}

Submit your manuscripts at

http://www.hindawi.com
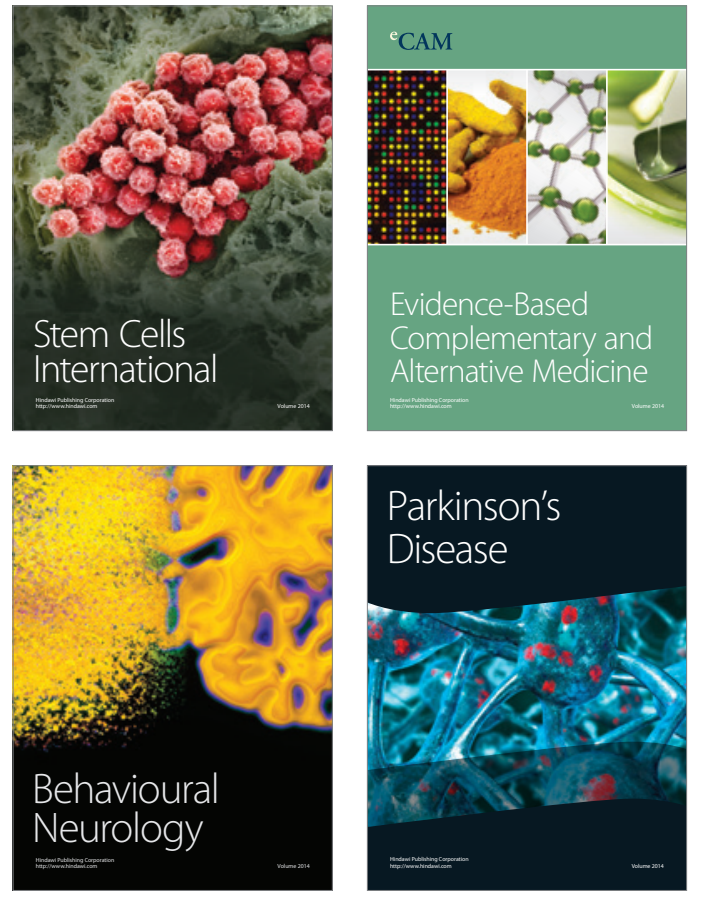
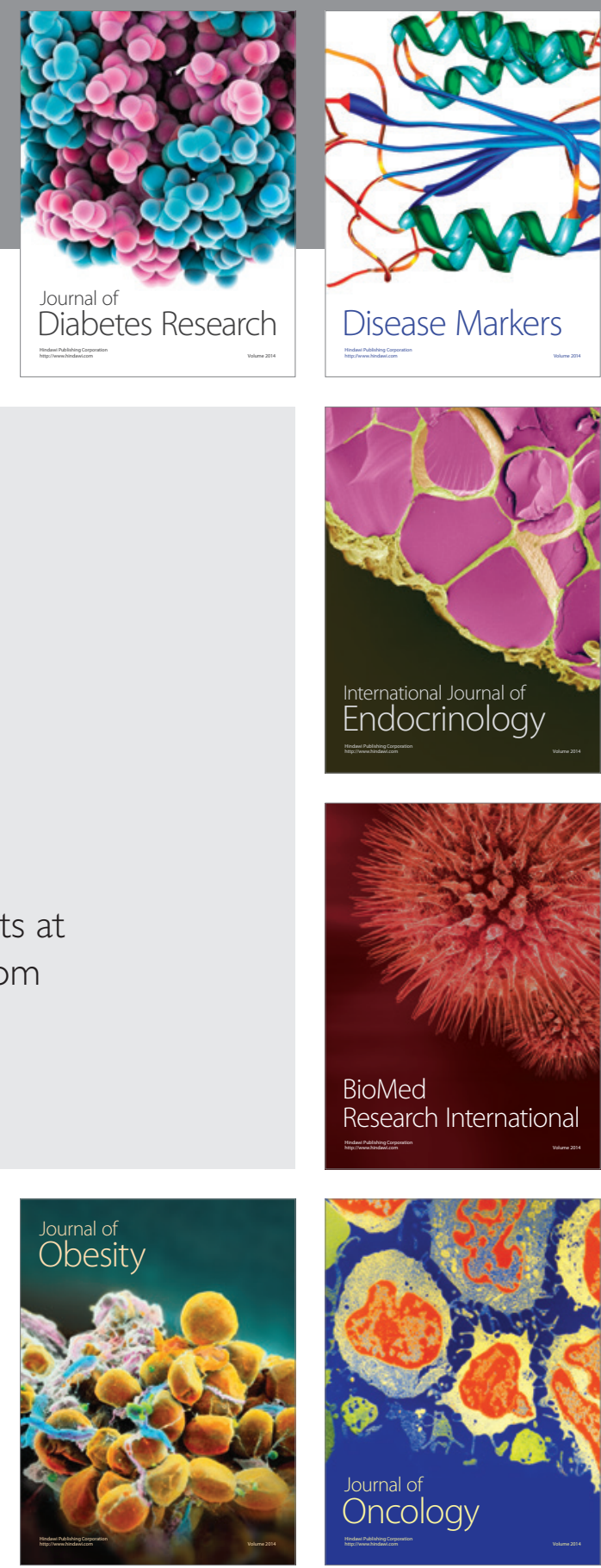

Disease Markers
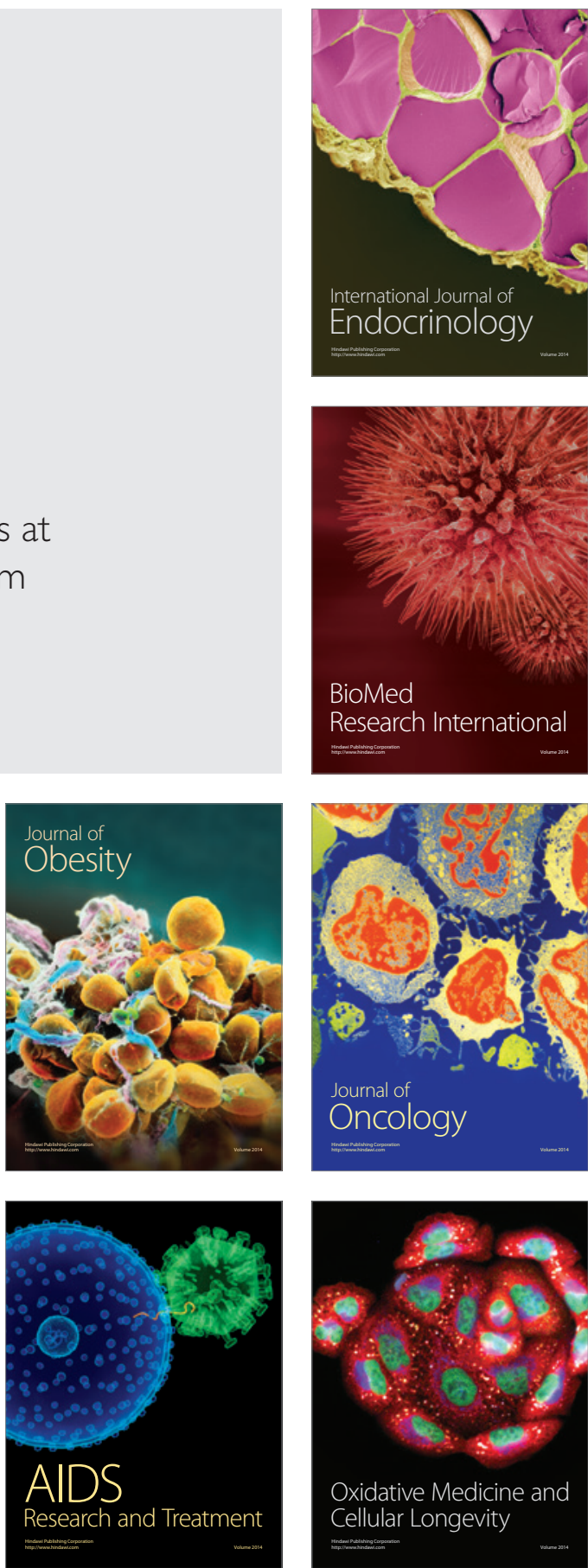